\title{
Advantages of Nanotechnology at the Solid-Liquid Interface
}

\author{
Andreas Stemmer*, Patrick Mesquida, and Nicola Naujoks
}

\begin{abstract}
Nanotechnology at the solid-liquid interface offers attractive prospects but also poses formidable challenges. Nature has evolved a vast range of functional nanostructures operating in the liquid environment of cells. Such natural nanostructures might be employed as building blocks for artificial nanodevices. To this end novel strategies for nanofabrication need to be developed by taking advantage of basic principles underlying nano-assembly in nature. Here we discuss one such technique, the electrostatically guided attachment of nanoparticles and proteins to localized charges deposited at the solid-liquid interface.
\end{abstract}

Keywords: Charge writing · Maskless microfabrication · Nanofabrication · Particle deposition

\section{Introduction}

Nature has evolved a vast range of functional nanostructures operating in cells to supply them with energy, generate force for motility, facilitate and control the transcription of DNA into RNA and the translation of the RNA code into proteins, to name just a few examples. From a technological viewpoint it appears attractive to explore how such biological nanomachines could be employed as functional building blocks in artificial nanodevices, e.g. supplying a medical implant with energy extracted and converted from the surrounding tissue or body fluid rather than a battery. Such biological nanostructures typically rely on a liquid environment of appropriate ionic strength to maintain structure and function. Since their coupling to the macroscopic world will most likely occur via some solid state substrate, nanotechnology almost inevitably has to focus on the solid-liquid interface. Clearly, the solid-liquid interface has been recognized as a crucial component for interaction with nanostructures long before the current enthusiasm for nanoscience

${ }^{*}$ Correspondence: Prof. Dr. A. Stemmer Nanotechnology Group

Swiss Federal Institute of Technology ETH Zentrum CLA

CH-8092 Zurich

Tel.: +411632 4572

Fax: +4116321278

E-Mail: stemmer@nano.mavt.ethz.ch

www.nanotechnology.ethz.ch and technology. Purification of proteins, for example, often takes place via chromatography, adsorption or desorption steps whereby a liquid phase containing the proteins interacts with a stationary solid phase. The degree of separation and purification achieved by these techniques depends on the strength of attraction between proteins and the solid phase. Similarly, electron microscopists imaging biomolecules realized that the structural integrity of their samples and, therefore, the relevance of their studies, critically depend on the interaction forces experienced by the molecules adsorbed from solution to the solid specimen support required for microscopy. Although the techniques mentioned in the above examples deal with nanostructures and rely on interaction forces acting over distances on the nanometer scale and below, they are not considered 'nanotechnology' because they lack a crucial component, namely localization and the control over it. Technologically, localization on the sub-micrometer scale is typically achieved by lithography. However, the well established routes known from semiconductor microfabrication are not designed to take advantage of direct targeting of nanostructures to specified locations as observed in living cells, rather they rely on the use of masks and additional process steps to shield areas where deposition should not take place. In the following we shall explore the feasibility and potential of directly targeting nanostructures to specified locations on a substrate by means of electrostatic guidance in solution.

\section{Electrostatic Targeting}

Electric fields have proven to be extremely powerful to transport and separate molecules by electrophoresis and the field from localized charges can also guide oppositely charged objects to attach at specific sites. On the macroscopic scale, this principle is exemplified by the well-known xerography [1] where the localized deposition of toner particles on paper is guided by electrostatic attraction. Of course, one might wonder whether the principle of xerography could be applied to nanostructures and biological objects, in particular since the latter typically require an ionic environment where electric charges get rapidly screened due to the short Debye lengths. Electrostatic targeting, therefore, could only be effective in the immediate vicinity of localized charges, i.e. over separations of a micrometer or less, but not over larger distances. The capability of guiding and attracting nanostructures over distances larger than a micrometer, however, is of importance to allow for solutions carrying moderately concentrated nanostructures to minimize the risk of coalescence by collision prior to the localized deposition. Interestingly, the conflicting requirements of (i) nanostructures being surrounded by an ionic liquid and (ii) electrostatic interaction acting over large distances can be reconciled by using a nonpolar solvent as transport medium [2]. 


\subsection{Transport Medium}

The range of the electrostatic interaction responsible for guiding and attracting nanostructures to localized electric charges in a solid substrate can be increased by replacing the aqueous solution with a nonpolar solvent such as heptane or a fluorocarbon liquid, i.e. by lowering the dielectric constant of the medium. Whereas nanostructures easily become charged in an aqueous environment, e.g. via dissociation of surface groups, the charging mechanism in a nonpolar medium is different. The phenomenological rule of Coehn [3] states that upon contact between two different dielectric materials the one with the higher dielectric constant will charge up positively. Thus silica nanoparticles $(\varepsilon=3.7)$ become positively charged when suspended in the fluorocarbon liquid FC-77 $(\varepsilon=1.86)$. Although initially observed for the solid-liquid interface Coehn's rule apparently also holds for the liquid-liquid interface, e.g. water droplets $(\varepsilon=80)$ emulsified in FC-77 charge up positively (Fig. 1), although the two charging mechanisms may not be identical. This charging effect is very useful for targeting nanoparticles suspended in water to localized charges deposited on a suitable substrate. The water droplets act as carriers for the suspended nanoparticles and provide the appropriate ionic environment required for their cargo. Relative to the surrounding nonpolar medium of lower dielectric constant the surface of the water droplets is positively charged and thus the droplets will be attracted by negative charges on the substrate.

\subsection{Charge Lithography}

Localized charges can be stored in a variety of materials commonly known as electrets. So far we have investigated silicon dioxide, poly(methylmetacrylate) (PMMA) and Teflon-like fluorocarbon layers for their charge storing properties. Teflon is known to offer excellent charge-storing properties but localized charges can be stored in all of the mentioned materials. Negative charges may be deposited by conventional electron beam lithography; however, in view of nanofabrication directly at the solid-liquid interface we opted for charge deposition via a conductive tip of an atomic force microscope (AFM). To this end we apply voltage pulses of defined length and height to highly doped silicon cantilevers operated in tapping mode. By scanning the AFM tip across the substrate arbitrary charge patterns can be created. Operating the AFM in contact mode while applying voltage pulses may lead to a damaged tip or sample surface. The dimensions of the charged area and the amount of de- posited charge are controlled by the height of the voltage pulses applied to the tip and the thickness of the fluorocarbon electret layer [4]. Higher voltage pulses lead to more charge deposited and a broader charge distribution. The pulse duration has little effect on the charging process provided the applied pulse lasts longer than a single tapping cycle. Therefore we may conclude that charge is only transferred during the first tap. Thin fluorocarbon layers of only a few ten nanometer thickness allow for higher resolution in charge writing than thick ones. The charge patterns deposited by AFM may be analyzed with the same tip by operating the AFM in the Kelvin probe force mode [5]. Apparent dot sizes as small as $100 \mathrm{~nm}$ in diameter are achieved with standard cantilever tips. Charges are conveniently written in air but are equally well deposited with the substrate and cantilever tip submerged in the insulating liquid transport medium.

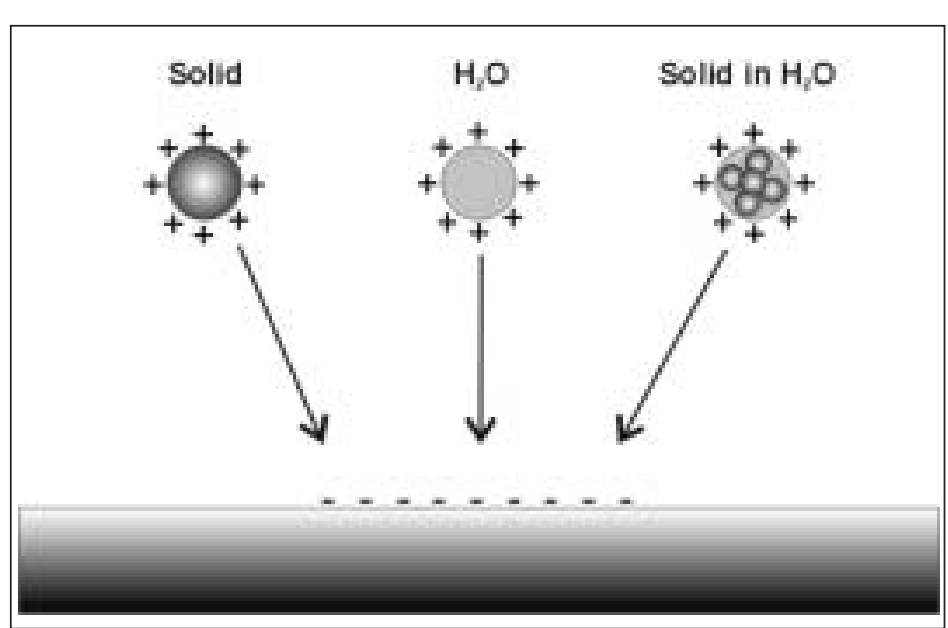

Fig. 1. Positive charging of solid materials and water droplets immersed in a nonpolar liquid of lower dielectric constant. Coulomb forces direct the charged particles and droplets to negative charges on the solid substrate.

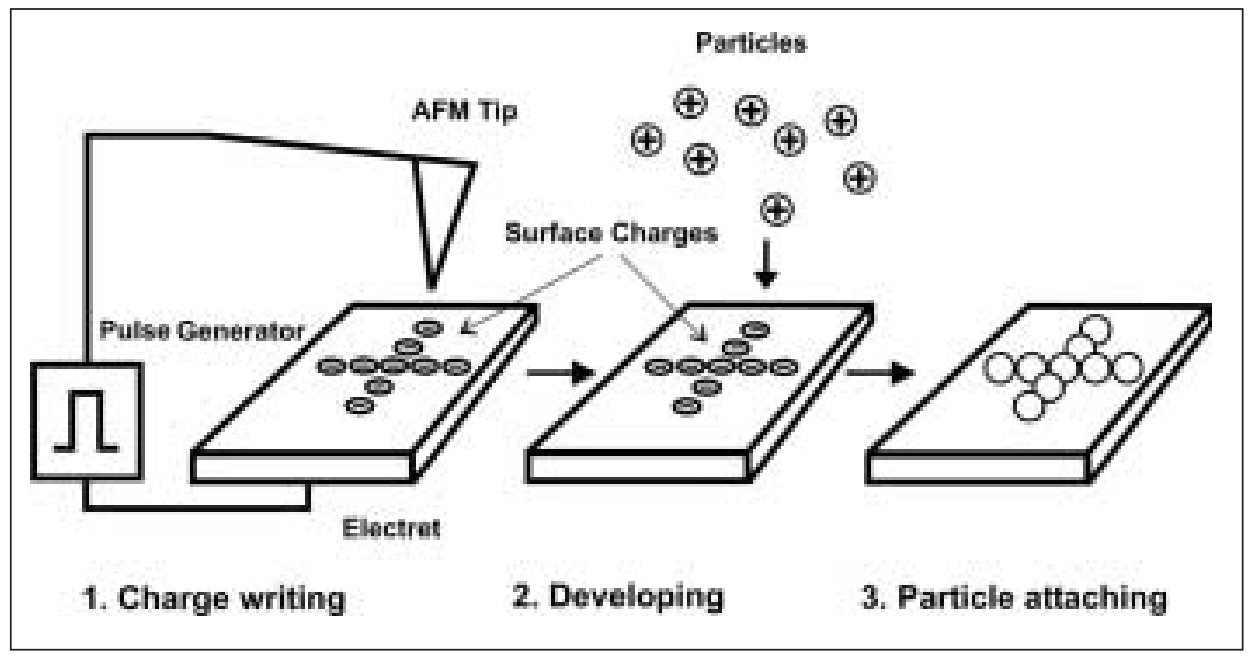

Fig. 2. Electrostatic targeting of nanoparticles to localized charge patterns on a solid substrate. The charge pattern is generated by applying voltage pulses to the conductive tip of an atomic force microscope. Charged particles of opposite polarity suspended in a nonpolar transfer liquid are attracted to the pattern and attach there.

\subsection{Particle Attachment}

Fig. 2 illustrates the three steps in selective particle attachment guided by electrocharges has been written onto the substrate, positively charged nanoparticles in the nonpattern of four lines with attached $20 \mathrm{~nm} \mathrm{Au}$ end four electrified lines of about 40-50 $\mu \mathrm{m}$ length and at a distance of about $10 \mu \mathrm{m}$ rom each other were written by applying a pulses of $-60 \mathrm{~V}$ height, 1 ms length ing the $A F M$ tip at a velocity of $10 \mu \mathrm{m} / \mathrm{s}$ ished Si-wafer coated with a $100 \mathrm{~nm}$ fluorocarbon layer produced by plasma enhanced chemical vapor deposition. The colloidal gold particles were initially suspended in water and had to be transferred static attraction. After a pattern of negative the where they firmly atto particles fabricated by this process. To this across the sample. The sample was a pol- 
into the nonpolar FC-77 liquid by sonication. This resulted in small positively charged water droplets containing gold nanoparticles. One might expect the citrate-stabilized gold nanoparticles carrying negative charges in water to be repelled by the negatively charged lines. However, the AFM topograph shown in Fig. 3 demonstrates that the particles firmly attach to the negatively charged lines in the substrate since the image was acquired after air-drying of the sample. Control experiments with positively charged lines showed no particle attachment.

Fig. 4 displays first results of guided deposition of proteins to electrified line patterns. The protein avidin, dissolved in phosphate buffered saline (PBS), was emulsified into the fluorocarbon liquid FC-77 by sonication. Thus, the PBS-droplets containing avidin molecules charged up positively and were attracted to negative charge patterns written into the substrate, a thin layer of PMMA spin-coated onto a polished Si-wafer.

\section{Prospects}

Nanotechnology at the solid-liquid interface is still in its infancy. Whereas imaging of delicate nanostructures submerged in liquid by AFM has matured into a reliable technique, their handling for controlled bottom-up assembly at specified locations still faces many challenges. Yet, the liquid environment also offers many unique oppor- tunities. State-of-the-art microfabrication technology, for instance, faces a tremendous 'dust-problem' and solves it via highly expensive clean-room facilities. Of course, small particles have a strong tendency to stick wherever they land and must be prevented from doing so, but living cells assemble their molecular machinery in an environment crowded by many components without interference. Cells achieve this task via specificity, i.e. components to be assembled need not only fit together mechanically but their fit must also be energetically favorable to remain stable. Interaction energies, however, are much easier tuned in liquid than in air or vacuum since, for instance, attractive van der Waals forces may be deliberately opposed by Coulomb forces. A liquid environment also serves as a convenient transport medium. Brownian motion does not appear to pose a problem since it can be easily biased by the application of electric fields as we have shown above. In fact, in nanotechnology at the solid-liquid interface Brownian motion and thermal energy in general provide a convenient means to differentiate between specific and non-specific interactions. The liquid environment also need not have a merely passive role. For instance, submicrometer electric circuit paths in aluminum thin films can be fabricated directly at the metal-water interface by the simple application of localized heat, e.g. by a focused low-power laser beam [6]. Here water provides a suitable electrochemical environment for the corrosion reaction and, in addition, serves as transport medium for removing the dissolved reaction products.

How much nanotechnology can we envisage at the solid-liquid interface? Looking at living cells probably a lot. The key issue is how to translate basic principles that evolved in nature into technologies accessible by today's means. Much knowledge on particular aspects of such basic principles has accumulated in separate scientific disciplines. The challenge for advancing nanotechnology at the solid-liquid interface, therefore, is not solely a technical one but equally well one of incorporating insights offered by a multitude of distinct disciplines.

Received: August 19, 2002

[1] J. Mort, 'The Anatomy of Xerography: Its Invention and Evolution', McFarland, London, 1989.

[2] (a) P. Mesquida, A. Stemmer, Adv. Mater. 2001, 13, 1395; (b) P. Mesquida, A. Stemmer, Microelectronic Engineering 2002, 61-62, 671.

[3] A. Coehn, U. Raydt, Annalen der Physik IV. Folge 1909, 30, 777.

[4] P. Mesquida, H.F. Knapp, A. Stemmer, Surf. Interface Anal. 2002, 33, 159.

[5] (a) M. Nonnenmacher, M.P. O'Boyle, H.K. Wickramasinghe, Appl. Phys. Lett. 1991, 58, 2921; (b) H.O. Jacobs, A. Stemmer, Surf. Interface Anal. 1999, 27, 361.

[6] D. Haefliger, A. Stemmer, Appl. Phys. A 2002, 74, 115 .

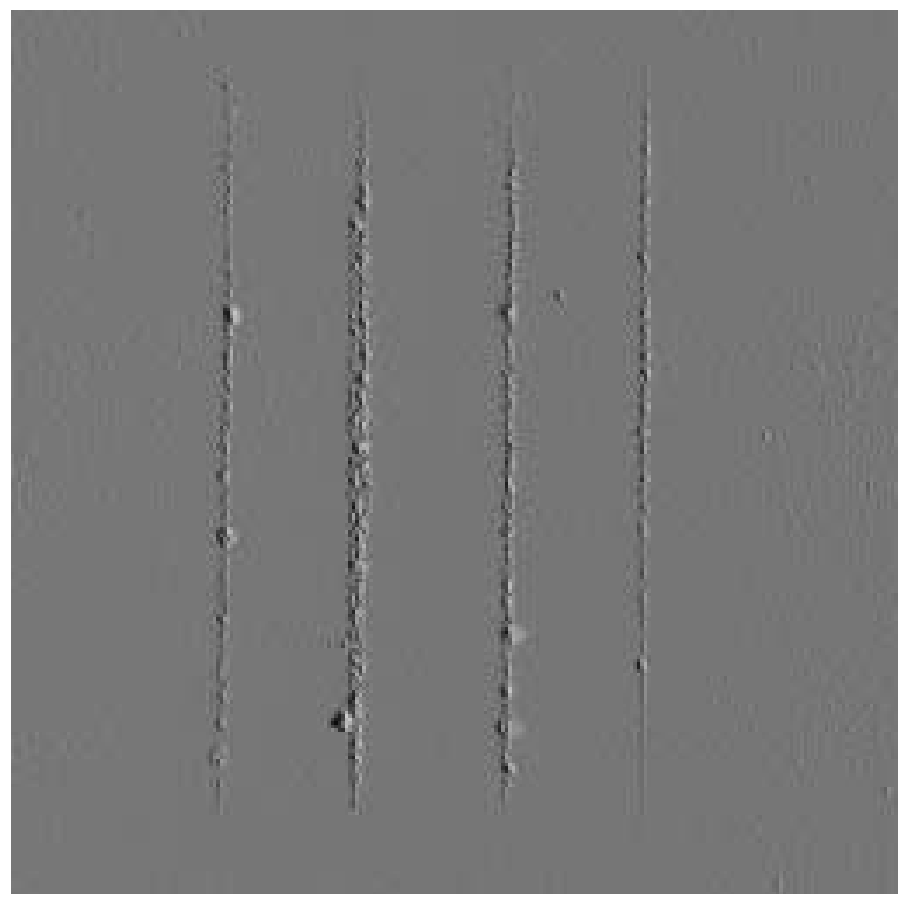

Fig. 3. Gold nanoparticles of $20 \mathrm{~nm}$ diameter attached to four negatively charged lines. Substrate is a polished Si-wafer coated with a $100 \mathrm{~nm}$ thick fluorocarbon layer. Image size is $60 \mu \mathrm{m} \times 60 \mu \mathrm{m}$.

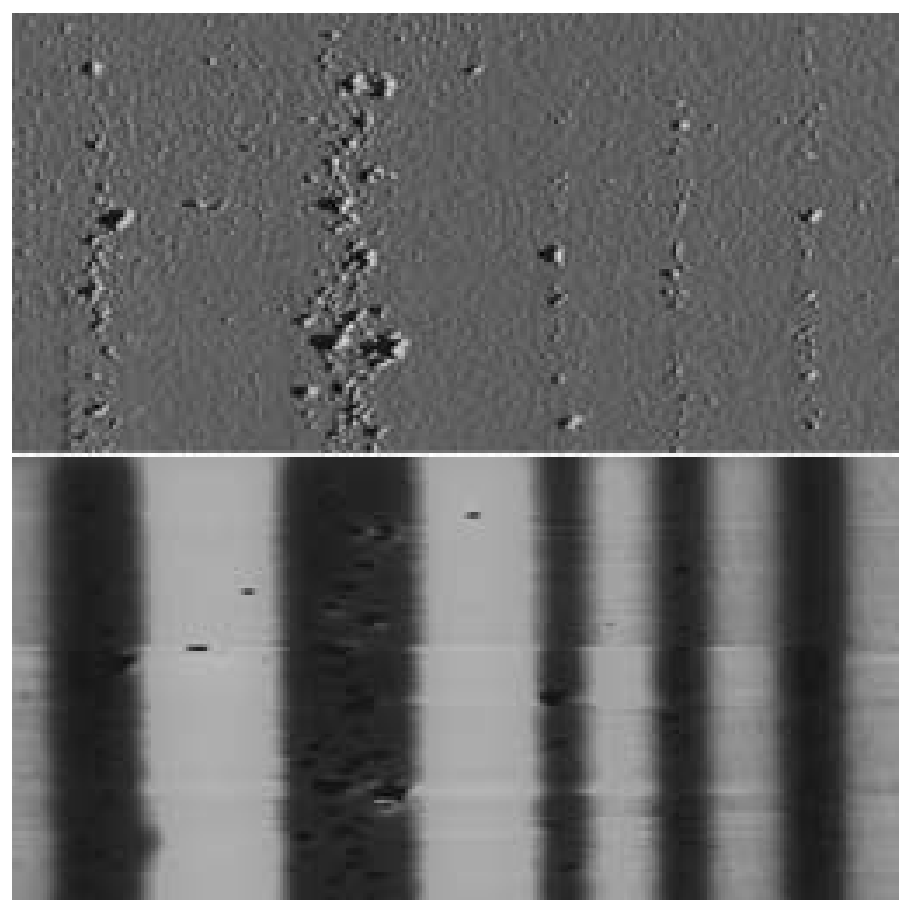

Fig. 4. The protein avidin deposited on negatively charged lines of different width. AFM amplitude image of the deposited protein aggregates (top) and Kelvin probe image (bottom) of the negatively charged lines. Substrate is a polished Si-wafer coated with a $150 \mathrm{~nm}$ thick PMMA layer. Image size is $40 \mu \mathrm{m} \times 20 \mu \mathrm{m}$. 